\title{
Updates in Targeted Sensory Reinnervation for Upper Limb Amputation
}

\author{
Jacqueline S. Hebert $\cdot$ Kate Elzinga $\cdot$ \\ K. Ming Chan · Jaret Olson $\cdot$ Michael Morhart
}

Published online: 23 January 2014

(C) Springer Science + Business Media New York 2014

\begin{abstract}
Advanced robotic devices capable of simulating the dexterous ability of the upper limb with an array of internal sensors have raised the enticing prospect of replacing the lost intricate functions of the arm following upper limb amputation. However, a large gap still exists in the application of this technology to the human user. In particular, the ability to provide physiologically relevant sensory feedback - to have the amputee feel the prosthetic hand as their own - has not yet been achieved. Although a number of different approaches are being investigated, targeted sensory reinnervation, a refinement of the original targeted muscle reinnervation procedure, is the most recent and promising development in the effort to create a
\end{abstract}

This article is part of the Topical Collection on Trauma-Related Prosthetic Surgery.

J. S. Hebert $(\bowtie)$

Division of Physical Medicine and Rehabilitation, Glenrose

Rehabilitation Hospital, University of Alberta, Rm 1239,

10230-111 Ave, Edmonton, AB T5G 0B7, Canada

e-mail: jhebert@ualberta.ca

K. Elzinga

Division of Plastic Surgery, University of Alberta, 2207-8210

111 St NW, Edmonton, AB T6G 2C7, Canada

\section{K. M. Chan}

Division of Physical Medicine and Rehabilitation, Centre for Neuroscience, 5005 Katz Group Centre, University of Alberta, Edmonton, AB T5R 2E1, Canada

J. Olson

Division of Plastic Surgery, University of Alberta, 82-8440 112

St NW, 2D3 WMC, Edmonton, AB T6G 2B7, Canada

M. Morhart

Division of Plastic Surgery, University of Alberta, 303 East

Tower, 14310111 Ave NW, Edmonton, AB T5M 3Z7, Canada functional human-machine interface with a closed loop sensory feedback system. This technique aims to reestablish hand sensation on the skin so that it can be readily accessed non-invasively during functional tasks. Recent efforts are being directed towards distributing hand maps widely on the stump without interference of sensations from the native area. In this article, we will review the surgical approaches that have been used for sensory reinnervation in upper arm amputation and compare the resultant outcomes and potential functional utility of the techniques.

Keywords Targeted sensory reinnervation - Targeted motor reinnervation · Upper limb amputation · Upper limb prosthesis $\cdot$ Sensory outcomes

\section{Introduction: Upper Limb Amputation-Prosthetic Advances and Challenges}

Despite major advances in engineered technology, proximal upper limb amputation remains one of the most difficult challenges for prosthetic replacement. Individuals with proximal levels of arm amputation have a higher rate of rejection of prostheses in comparison to more distal levels of amputation [1, 2]. Reasons for rejection are widely varied. The main concerns from myoelectric users that limit use of the prosthesis include poor durability, poor dexterity, and lack of sensory feedback [3].

In response to these concerns, artificial limbs with up to 22 degrees of freedom have been developed in an attempt to design a natural limb replacement device with greater function [4-6]. However, despite the existence of multifunctional prosthetic limbs and efforts to deploy these into clinical practice, challenges with implementation include 
difficulties attaching the device to the patient, insufficient motor control strategies to control the additional degrees of freedom, and lack of sensory feedback from the device to the human operator. Advances are being made with novel socket designs to improve comfort and suspension [7], and there is ongoing research into percutaneous skeletal attachment to allow direct connection of the prosthesis to the skeletal system [8]. Emerging motor control strategies such as pattern recognition algorithms are promising in the potential ability to control multiple actions of the prosthetic limb [9]. However, designing a method to restore natural sensation from the prosthetic limb is still an unsolved challenge in the effort to restore dexterous hand function following upper limb loss.

Developing a neural human-machine interface that receives and decodes sensory information is a difficult task. The importance of "natural, physiologic sensation" cannot be overlooked when attempting to restore sensory function to an artificial limb. Various types of sensory feedback from prosthetic devices have been trialed in the past [10, 11-] but with no success in clinical translation or long-term usage. This is likely because substitution methods had to be used-that is, the amputee would have to be "trained" to understand that an unnatural (or non-physiologic) stimulus meant that something of importance was happening to the prosthesis. This form of sensory substitution can work in controlled settings; however, it has not lead to long-term adoption. The basis for rejection of the feedback device may be that it does not tap into natural sensory mechanisms or provide a percept that enhances the feeling that the prosthesis belongs to the individual as their own hand.

\section{Neural-Machine Interfaces for Upper Limb Amputation}

Neural interfaces in both the peripheral and central nervous system have been developed as a method to provide sensory feedback. Peripheral nerve stimulation, as a mechanism for restoring sensory information from the prosthetic device to the amputee, has been investigated for decades [12]. Over the last few years, the feasibility of using direct neural interfaces to stimulate peripheral afferent nerves has progressed from animal to human studies, with evidence that the sensory input provided allows modulation of motor control [13, 14], but with variable reports on the naturalness of the sensations elicited by electrical stimulation [11•]. Despite several types of neural interfaces available, current technological limitations of the implanted electrodes preclude long term use [15]. Cortical approaches for sensory restoration have been examined in animal models [16]. More recently, brain machine interfacing has been examined for motor control in human subjects [17], but the role of somatosensory cortex stimulation has not yet been explored in humans.

For these approaches, the long term viability of the implanted electrodes is the main challenge, and the potential unnatural quality of the sensation elicited by the peripheral nerve stimulation needs to be addressed [11•]. Therefore, although there is interesting potential for the use of peripheral and central neural interfaces for both motor and sensory control, further research is required in human subjects to define their clinical applicability for long-term use.

\section{Targeted Reinnervation Surgery for Upper Limb Amputation}

Of the approaches developed to overcome the humanmachine interface challenge, the most clinically accessible, viable, and currently used technique is that of targeted reinnervation (TR), pioneered by Kuiken et al. [18] at the Rehabilitation Institute of Chicago and Northwestern University. Kuiken's early work with hyper-reinnervation demonstrated the robust ability of amputated nerves to reinnervate new muscle sites and provide physiologically useful motor units for myoelectric control. The translation of this technique to the human model was demonstrated with initial case reports for shoulder level disarticulation [19-21] and transhumeral subjects [22, 23].

The TR procedure is now an established clinical treatment method for improving motor control sites for proximal upper limb amputation. Typically performed as a secondary revision procedure, there is an extremely high rate of muscle reinnervation success (reported as greater than $90 \%$ ) and ability to use these sites for myoelectric control [19, 23]. The successful outcome of TR is reflected not only in the robust muscle reinnervation observed following surgery, but also in the application of new prosthetic technology. Patients are able to operate multiple degrees of freedom of advanced prosthetic devices with increased ease following TR surgery and appropriate rehabilitation [21, 24-26].

The benefits of the TR procedure extend beyond motor reinnervation. Some of the amputees that underwent the TR procedure were found to have restoration of sensation in the missing hand and arm on the skin overlying the areas of muscle reinnervation $[21,27 \bullet \bullet]$. When they were touched on the skin, it felt like they were actually being touched on the missing limb, suggesting that cutaneous reinnervation by sensory afferents was occurring in addition to the motor reinnervation. This led to the intriguing possibility of linking an area of reinnervated skin that "feels" like the amputee's own hand to a sensory input device matched to the prosthetic hand. In this way, a physiologically natural 
and appropriate avenue of sensory feedback might be restored.

\section{Sensory Reinnervation Approaches}

Since the initial observations of sensory changes associated with TR, there have been three main surgical techniques associated with sensory reinnervation: (1) traditional TR with motor branch coaptation and associated denervation of overlying skin to allow sensory afferents to reinervate the skin; (2) TR with the addition of end-to-side coaptation of a cutaneous sensory nerve to a main nerve trunk, and (3) an end-to-end targeted fascicular sensory reinnervation technique using intra-operative nerve stimulation to aid in fascicle selection. Each of these techniques will be reviewed in turn.

\section{Traditional TR}

With this technique, a segment of skin near or overlying the TMR (targeted muscle reinnervation) site is denervated and the regenerating afferent nerve fibres from the residual hand nerves are enabled to reinnervate this area of skin. [21]

The original TR procedure describes end-to-end coaptation of sectioned median, ulnar, and radial nerve trunks to discrete motor nerve branches of target muscles. For the transhumeral residual limb, this includes coapting the median nerve trunk to the medial head of biceps, the distal radial nerve trunk to the lateral head of triceps, and, when possible, the ulnar nerve trunk to the brachialis. The reinnervated muscle sites provide natural hand open and close signals. The musculocutaneous nerve innervating the lateral head of the biceps and the proximal radial nerve innervating the long head of the triceps are left intact for the elbow flexion and extension signals [23].

For the shoulder disarticulation amputee, the pectoralis major is sectioned into three parts: the clavicular head, an upper sternal head, and a lower sternal head. The pectoralis minor is divided from its origin, rotated laterally from beneath the pectoralis major, and secured in the mid-axillary line to allow the muscle to lie directly below the skin. These four newly created muscle segments are then denervated from their original innervating nerves. Coaptation is performed from the musculocutaneous, median, radial, and ulnar nerve trunks to the clavicular head, upper sternal head, and lower sternal head of the pectoralis major and to the pectoralis minor, respectively, to allow reinnervation of these muscles by the selected nerves and to provide the hand and elbow control signals [19, 20]. Alternative target muscles that may be used include the serratus anterior, trapezius, and latissimus dorsi muscles.
As part of the surgical technique, the skin overlying the anticipated motor points was thinned of subcutaneous tissue to reduce electrode interference in the first TMR case $[19,22,28]$. This resulted in local denervation of the skin, which then allowed for competitive reinnervation of afferent nerve fibers. In particular, the sensory afferents of the large mixed nerve trunks that were redirected to the motor points of the muscles competitively reinnervated the overlying denervated skin [19, 27••]. The denervation of the overlying skin was essential to allow the regeneration of hand afferents to the newly vacant cutaneous receptors, although in some transhumeral subjects the intercostobrachial cutaneous nerve has been intentionally cut in order to denervate the skin (Kuiken, personal communication, 2013).

Within 4-6 months following the surgery, corresponding to the expected rate of reinnervation, sensations referred to the missing limb may develop when the skin overlying the reinnervated muscle is touched. However, rather than correlating to the precise somatotopic map of each transferred nerve, the referred sensations are typically variable and intermixed, including native anatomic skin sensation [27••]. Although near normal thresholds are developed in those areas with restored hand sensation, the variability of the somatotopy potentially limits the predictability and reliability of using this approach specifically for harnessing a sensory feedback access point.

\section{Cutaneous Nerve End-to-Side Targeted Sensory Reinnervation}

Shortly after these initial findings, Kuiken et al. [21] explored a modification of the TR procedure. The modification involved adding two end-to-side sensory nerve transfers during the TR procedure in a proximal transhumeral (functional shoulder disarticulation) amputee. The supraclavicular cutaneous nerve was cut and the distal segment was coapted to the side of the ulnar nerve. The intercostobrachial cutaneous nerve was also cut and then coapted to the side of the median nerve. The proximal end of the sensory nerves were mobilized superiorly in an effort to prevent reinnervation of the chest skin. The subcutaneous fat was thinned, but not completely removed, to improve the surface muscle signal without causing contour deformity of the overlying skin [21].

Post operatively, the insensate areas corresponded to the expected cutaneous nerve distributions. By 6 months, somewhat surprisingly, the anterior chest skin was reinnervated by both median and ulnar afferents. There were a few primary spots where sensation in just one digit was felt, but in most areas a mixture of digit and native chest sensations were elicited. Several modalities of cutaneous sensation were restored, including sharp/dull and warm/ 
cold sensation, graded pressure, and vibration with near normal thresholds of perception. However, the sensations were not completely "normalized," in that touch of the target skin was associated with a tingling sensation rather than normal pressure sensation [21]. As with the initial TR technique, there did not appear to be specific somatotopy with the referred-hand sensations [27••].

The sensory somatotopic organisation that developed differed from what we expected. In surgery, the distal segment of the intercostobrachial nerve was transferred to the median nerve and median sensation was anticipated on the lateral chest. Only a faint median percept developed, probably because the skin innervated by the intercostobrachial nerve was amputated with the arm leaving nothing for the median afferents to reinnervate. The distal segment of the supraclavicular nerve was transferred to the ulnar nerve, and ulnar sensation alone was expected on the anterior chest. However, a strong percept and large area of median nerve reinnervation were noted. The robustness of the median nerve sensory reinnervation was surprising. The median nerve afferents had to regenerate through the pectoralis major muscle and through a layer of subcutaneous tissue that was more than $1 \mathrm{~cm}$ thick while in competition with the regenerating ulnar afferents. Further study is clearly needed to better understand what guides, promotes, or impedes sensory axon regeneration. [1]

With this end-to-side technique, there was improvement in control of the sensory reinnervation territory, but not in exclusivity for the recipient nerve as evidenced by the ability of the competing median nerve afferents to find their way to the desired site of ulnar nerve reinnervation. The partial control of the direction of reinnervation was highly promising in that there was a discrete region of transfer sensation created by afferent axons reinnervating appropriate end organs in the skin with close to normal perception thresholds for touch, temperature, pain, and vibration [27••, 29].

Similar experiences have been observed in transhumeral subjects with end-to-side anastomosis of the intercostobrachial cutaneous nerve to the median nerve trunk, with variable restoration of hand sensations in the distal arm region not restricted to the median nerve hand map (Kuiken, personal communication, 2013).

\section{Sensory Fascicle End-to-End Targeted Sensory Reinnervation}

The above observations motivated the search for a method of improving control over the sensory reinnervation process, to harness more reliably the restoration of the hand map on cutaneous target areas of the residual limb. This procedure uses somatosensory evoked potentials (SSEP) to assess more finely the sensory content of specific fascicles of the main reinnervating nerves, and identification of target cutaneous nerves remote from the anticipated motor sites [30••].

Detailed guidelines for the use of SSEP for intraoperative monitoring have been published by the American Society of Neurophysiological Monitoring [31•]. Several authors have used SSEPs of the main nerve trunks to assess the status of the peripheral nerves and brachial plexus to avoid injury during upper limb surgery [32-34], to aid in the differentiation of nervous tissue from fibrous tissue during dissection in a scarred operative field [35], and to assist with identification of healthy median nerve [36]. Less commonly, electrical stimulation has been used to identify sensory fascicles in peripheral nerves $[37,38]$, but in a time consuming manner relying on the cooperation of the patient who is required to localize the stimulation after being awoken intra-operatively ("awake stimulation").

The sensory fascicle end-to-end TR technique developed by the authors uses electrical stimulation in a different manner. The median and ulnar nerves are known to be mixed nerves with distinct fascicular arrangements, with the majority of the sensory fibers dedicated to sensation of the digits [39, 40]. Use of intra-operative SSEPs by recording over the somatosensory cortex allows efficient inter-operative identification of fascicles with high sensory content from the median and ulnar nerves by individually stimulating separated fascicles after intrafascicular dissection. Once a predominantly sensory fascicle is identified and coapted to a cutaneous sensory target nerve, the remainder of the main nerve is directed to the motor branch of the target muscle.

The choice of target cutaneous nerves depends on the available residual limb length and should be identified preoperatively as areas with normal sensory thresholds. For the transhumeral level, the skin over the lateral upper arm supplied by the cutaneous branch of the axillary nerve (C5-6) [41, 42], and the intercostobrachial nerve (T2) [42, 43] supplying the medial proximal arm are candidate target sensory areas. These nerves are chosen for their remoteness from the anticipated motor sites in the transhumeral limb, which are the medial and lateral muscle bellies of the biceps and triceps (Fig. 1).

During the TR procedure, the cutaneous sensory target nerves are identified and tagged as part of the initial dissection. Once the main median nerve trunk is identified, the whole nerve trunk is stimulated supramaximally to determine the maximum SSEP amplitude. Then intrafascicular dissection of the main nerve trunk is undertaken, initially a short exposure to separate and lift out two to three single fascicles. Each individual fascicle is in turn lifted away 


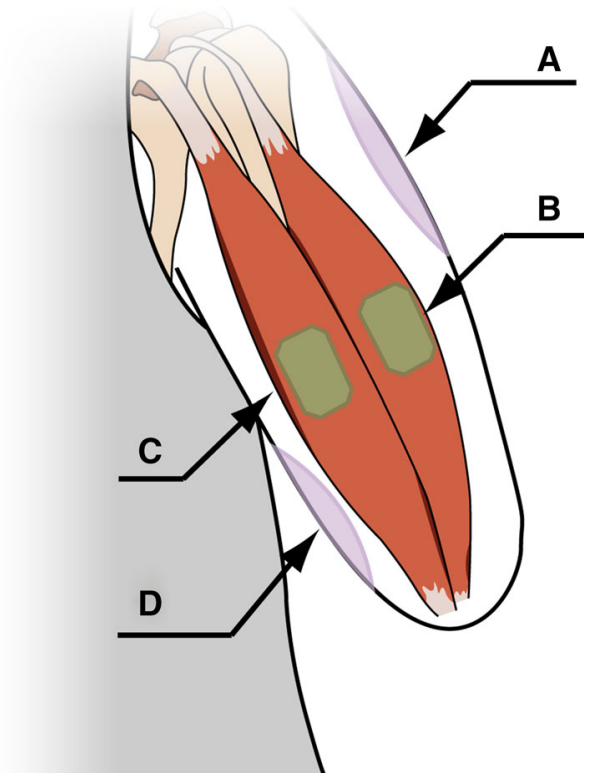

Fig. 1 Schematic of target cutaneous sites $(a, d)$ in relation to anticipated motor control sites $(b, c)$ in the transhumeral limb

from the nerve trunk and stimulated with a pair of silverball hook electrodes (Fig. 2). The SSEP for each individual fascicle is compared with the main nerve trunk SSEP. The fascicle with the largest relative SSEP is chosen for transfer to the cutaneous sensory branch target. The chosen fascicle is dissected from the main nerve trunk to allow sufficient length to reach the target sensory nerve. The cutaneous sensory nerve is sectioned as close to the entry point of the skin as feasible to minimize the time of regeneration. Once end-to-end coaptation of the cutaneous sensory branch to the selected sensory fascicle is performed, the remainder of the main nerve trunk is used for the standard motor reinnervation procedure.

This does not compromise the motor reinnervation because a large innervation ratio still exists. The resulting sensory reinnervation is expected to be more precisely controlled than the end-to-side technique [44]. Post-operatively, areas of insensitivity should correspond to the cutaneous nerve territories, and recovery of digit sensation should begin within 4-6 months. In the case study reported, this technique resulted in discrete exclusive sensory patches of the ulnar and median nerve hand maps in the designated territories, with no overlap [30••]. Interestingly, there was also no competitive reinnervation of denervated skin over the target muscles, where normal sensation of the arm skin was retained.

Similar end-to-end fascicular procedures have not been performed for the shoulder disarticulation level. However, the skin over the clavicle and superolateral aspect of the pectoralis major supplied by the supraclavicular nerve (C3-4) may be the easiest nerve to access given the

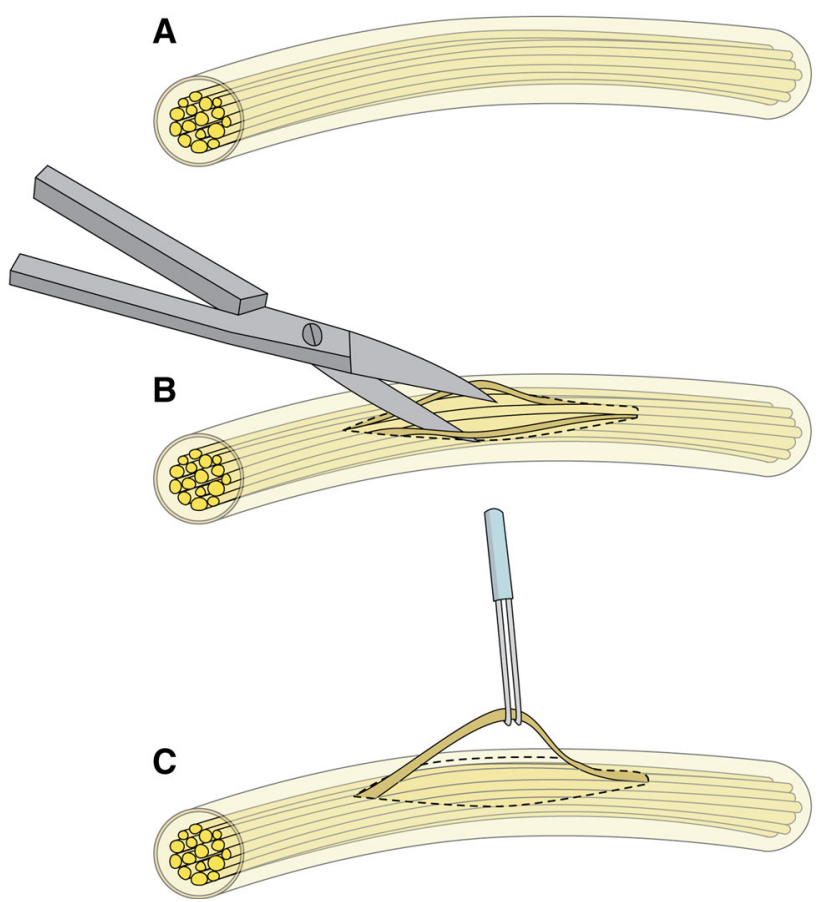

Fig. 2 Schematic of intrafascicular dissection and individual fascicle stimulation. a Entire nerve trunk; b intrafascicular blunt dissection; c single fascicle being stimulated with silverball hook electrode

surgical approach through an anterior supraclavicular incision [45]. Adding the intrafascicular technique to the end-to-side coaptation may assist with the specificity of reinnervation, but there is still potential interference of the underlying pectoralis muscle contraction. The proximal branch of the intercostobrachial cutaneous nerve could also be used as a target for sensory reinnervation at a shoulder disarticulation level, as the axillary skin area would be expected to cover the lateral aspect of the trunk following the amputation. Intercostal nerves (T1-12) supply the skin of the chest and abdomen in a segmental fashion [46] and these may be potential cutaneous targets, but further investigation is needed to investigate the feasibility of this approach.

\section{Discussion: Sensory Reinnervation Outcomes}

Given the small group of subjects with sensory reinnervation and noted individual variation, it is difficult to compare directly these three techniques with respect to potential effect on functional outcome. Outcomes reported include referred hand sensation mapping, sensory modality thresholds, and application of haptic devices for feedback in a virtual environment or with motor control of an experimental device. The results reported in the literature can be used as an illustration of the physiologic and preliminary functional outcomes for each approach. 
Physiologic Outcomes

A wide range of sensory modalities has been restored in subjects with sensory reinnervation. This includes near normal touch, temperature, and pain sensation as determined by response to electrical stimulation [27••] and vibration thresholds [29], suggesting the regeneration of large mechanoreceptors as well as small diameter temperature and pain afferents. This extends to the ability to detect gradations in force at near normal levels [47]. Reinnervated skin grating orientation thresholds have been shown to be normal in comparison to control skin areas, and point localization thresholds increased [48], which may reflect preservation of the large central processing region for the hand afferents. However, in addition to touch and pressure, some patients feel a dysesthesia or sensation that is altered in quality [21]. This outcome, which is common in other procedures that disrupt the sensory nerves, cannot yet be predicted or controlled.

In subjects having had traditional TR or end-to-side coaptation, there has been significant intermingling of the referred hand sensation with that of the native chest.

In both patients there were distinct areas of the chest where the regenerated arm sensation overlapped with the native chest sensation. The resulting sensation was not confusing to either patient; they simply reported feeling as though they were being touched in two different areas at once. [27••]

A large variability has been seen with respect to the somatotopy of the resulting hand map in the traditional TR procedure and the end-to-side coaptations [21, 27••]. In general, there appears to be little control over which afferent fibers regenerate from the transferred nerves, and both subjects demonstrated more than one referred nerve distribution within their reinnervated territory [27••]. In contrast, in the subject with the fascicular end-to-end technique, there is a fairly dense representation of only the volar aspect of the thumb, index, and middle fingers in the median reinnervated skin [30••]. The degree of disorganization of the hand map may, therefore, be reduced by the end-to-end fascicular technique, as a single fascicle with high sensory content is more likely to be closely associated somatotopically [39, 49] compared to the afferent fibers of the entire nerve trunk competitively reinnervating the skin.

With respect to long term maintenance of sensory reinnervation, all subjects have been followed for over 1 year after amputation, and sensation has been stable for greater than 5 years post amputation in the first subject reported $[27 \bullet \bullet]$. Therefore, it seems that the sensory pathways have been robust and are able to endure despite the lack of functional usage.
Functional Experiments: Haptic Devices

The ability to detect gradations in force using a haptic device has been tested with and without link to a functional task. In a force discrimination trial, the subject with sensation restored with the end-to-side transfer of the supraclavicular nerve was limited by the lack of available indentable skin in the region under the clavicle [47]. Those experiments did show an ability to detect force gradation, but this was not linked to a functional task.

In a virtual environment haptic feedback trial, somatotopically matched haptic feedback was able to enhance grip force control, but not task completion speed in subjects with the traditional and end-to-side sensory reinnervation [50]. Interesting findings in this study were that performance with single modality feedback was better than with multiple modalities, there was significant intra-subject variation, and not all types of stimulation were tolerated by both subjects. In addition, muscle site contraction interfered with the subject's ability to sense the tactile feedback, and could not be performed simultaneously. This may be the best rationale for the need to separate the target sensory areas from the underlying motor sites. It was also noted that the tactor did not deliver a "realistic" sensation. It is uncertain whether this was due to the use of the virtual environment versus a real world functional control task, or due to the alternating control/sense technique used by the subjects.

In the functional experiment with the subject with the fascicular end-to-end technique, a myoelectric robotic training tool that the subject controlled with native and reinnervated muscle sites was used as the testing platform [51]. This was done simultaneous to haptic touch and pressure feedback linked to the terminal gripper. It was noted that although the gripper did not resemble a typical prosthetic hand, the subject reported that the sensation of touching the ball with his own hand was quite strong. The subject was able to determine differences between stiffness of objects and was able to use the distinct somatotopy of two discretely innervated areas (median index digit and ulnar small digit) for object size identification [30••]. The limitations of this study were that no control sites were used as comparison, and the inability to prove physiologic matching other than by patient report. However the results did demonstrate the ability to have dual flow of information (motor and sensory) in a simultaneous fashion within the residual limb.

It will be important in future sensory feedback experiments on sensory reinnervation that both modality matching and somatotopic matching be present, along with simultaneous motor control. This is reinforced by prior work that has shown the importance of sensorimotor coupling to intuitive control [52]. 


\section{Conclusions and Future Directions}

There is a complex interplay between surgical approach, physiological outcome, and engineered devices to create a successful human-machine interface with sensorimotor function. The surgical goal is to maximize the control, spatial discrimination and specificity of the hand map with the widest range of discrete modality sensation.

Microsurgical approaches and application of peripheral nerve repair techniques have greatly advanced the understanding of reconstruction of the amputated residual limb, including the use of fascicular techniques [53•]. It is now possible to rebuild and reshape motor and sensory neural interfaces, in addition to the bones and muscle attachments. This is an exciting advancement in the creation of a functional human-machine interface, with dual flow of neural information. However, these techniques are still in their infancy and further work is needed to optimize the interface with newly reinnervated skin and sensory organs. Restoration of the rich median and ulnar hand sensory maps is a start-it provides a rich template on which to build the right interfaces. The sensory percepts studied in the TR literature focus on cutaneous sensation, but other modalities such as kinesthesia may be more reliably elicited in the skeletal muscle tendon unit, which may not require cutaneous reinnervation [54]. In addition, advancement in the longevity of neural interfaces may one day allow us to bypass the cutaneous end organ and go directly to the peripheral nerve interface [15], in which case the selection of peripheral fascicles may be facilitated by SSEP fascicle selection.

Lastly, the importance of the rehabilitation and recovery process needs further exploration. Following the TR sensory procedure, it can take 4-8 months for initial sensation and up to 1 year for stable reinnervation to occur. We have yet to apply specific sensory reeducation techniques to sensory reinnervation subjects. Training sensory recovery and strengthening the embodiment response [55•] may improve the naturalness of sensation, cortical reintegration, and ultimate acceptance of the sensory input from the prosthetic device.

The quest to restore sensation from a prosthetic limb is part of a larger line of research to restore natural control and usage of the device. Motor control strategies are equally as important as restoring sensation, as only with the capacity to control dexterous hand motions can sensory feedback be fully utilized. It should be noted that currently, although there is no commercial product to provide an integrated sensory feedback device within a prosthetic socket, research is ongoing in this area. Several haptic devices have been released or are in development, and socket integration is being actively explored. Ongoing research into creating wearable, integrated sensory feedback sockets with prostheses is required. Using the neural interface of TR as a substrate may allow us to integrate this next level of technology.

Acknowledgments The authors wish to thank Dr. Todd Kuiken for valuable input on the manuscript, and Michael (Rory) Dawson for figure creation.

\section{Compliance with Ethics Guidelines}

Conflict of Interest Jacqueline S. Hebert, Kate Elzinga, K. Ming Chan, Jaret Olson, and Michael Morhart declare that they have no conflict of interest.

Human and Animal Rights and Informed Consent This article does not contain any studies with human or animal subjects performed by any of the authors.

\section{References}

References of interest which have been published recently are noted as:

- Of importance

•• Of major importance

1. Wright TW, Hagen AD, Wood MB. Prosthetic usage in major upper extremity amputations. J Hand Surg Am. 1995;20(4): 619-22.

2. Biddiss E, Chau T. Upper-limb prosthetics: critical factors in device abandonment. Am J Phys Med Rehabil. 2007;86(12): 977-87.

3. Biddiss E, Beaton D, Chau T. Consumer design priorities for upper limb prosthetics. Disabil Rehabil Assist Technol. 2007;2(6):346-57.

4. Resnik L. Development and testing of new upper-limb prosthetic devices: research designs for usability testing. J Rehabil Res Dev. 2011;48(6):697-706.

5. Harris A, Katyal K, Para M, Thomas J. Revolutionizing prosthetics software technology. 2011 IEEE International Conference on Systems, Man and Cybernetics. 2011. doi:10.1109/ICSMC. 2011.6084102.

6. Armiger RS, Tenore FV, Katyal KD, et al. Enabling closed-loop control of the modular prosthetic limb through haptic feedback. Johns Hopkins APL Tech Dig. 2013;31(4):345-53.

7. Moran CW. Revolutionizing prosthetics 2009 modular prosthetic limb-body interface: overview of the prosthetic socket development. Johns Hopkins APL Tech Dig. 2011;30(3):240-9.

8. Jönsson S, Caine-Winterberger K, Brånemark R. Osseointegration amputation prostheses on the upper limbs: methods, prosthetics and rehabilitation. Prosthet Orthot Int. 2011;35(2): 190-200

9. Scheme E, Englehart K. Training strategies for mitigating the effect of proportional control on classification in pattern recognition based myoelectric control. J Prosthet Orthot. 2013;25(2): 76-83.

10. Lundborg G, Rosén B. Sensory substitution in prosthetics. Hand Clin. 2001;17(3):481-8.

11. - Antfolk C, D’Alonzo M, Rosén B, et al. Sensory feedback in upper limb prosthetics. Expert Rev Med Devices. 2013;10(1): 45-54. This paper reviews strategies for sensory feedback in 
upper limb amputation, focusing on sensory substitution and direct neural stimulation.

12. Riso RR. Strategies for providing upper extremity amputees with tactile and hand position feedback-moving closer to the bionic arm. Technol Health Care. 1999;7(6):401-9.

13. Dhillon GS, Horch KW. Direct neural sensory feedback and control of a prosthetic arm. IEEE Trans Neural Syst Rehabil Eng. 2005;13(4):468-72.

14. Horch KW, Meek S, Taylor TG, Hutchinson DT. Object discrimination with an artificial hand using electrical stimulation of peripheral tactile and proprioceptive pathways with intrafascicular electrodes. IEEE Trans Neural Syst Rehabil Eng. 2011;19(5): 483-9.

15. Micera S, Citi L, Rigosa J, et al. Decoding information from neural signals recorded using intraneural electrodes: toward the development of a neurocontrolled hand prosthesis. In: Proceedings of the IEEE. 2010. doi:10.1109/JPROC.2009.2038726.

16. Berg JA, Dammann JF III, Tenore FV, et al. Behavioral demonstration of a somatosensory neuroprosthesis. IEEE Trans Neural Syst Rehabil Eng. 2013;21(3):500-7.

17. Chase SM, Kass RE, Schwartz AB. Behavioral and neural correlates of visuomotor adaptation observed through a brain-computer interface in primary motor cortex. J Neurophysiol. 2012; 108(2):624-44.

18. Kuiken TA, Childress DS, Rymer WZ. The hyper-reinnervation of rat skeletal muscle. Brain Res. 1995;676(1):113-23.

19. Kuiken TA, Dumanian GA, Lipschutz RD, et al. The use of targeted muscle reinnervation for improved myoelectric prosthesis control in a bilateral shoulder disarticulation amputee. Prosthet Orthot Int. 2004;28(3):245-53.

20. Hijjawi JB, Kuiken TA, Lipschutz RD, et al. Improved myoelectric prosthesis control accomplished using multiple nerve transfers. Plast Reconstr Surg. 2006;118(7):1573-8.

21. Kuiken TA, Miller LA, Lipschutz RD, et al. Targeted reinnervation for enhanced prosthetic arm function in a woman with a proximal amputation: a case study. Lancet. 2007;369(9559):371-80.

22. O'Shaughnessy KD, Dumanian GA, Lipschutz RD. Targeted reinnervation to improve prosthesis control in transhumeral amputees. A report of three cases. J Bone Joint Surg Am. 2008;90(2):393-400.

23. Dumanian GA, Ko JH, O'Shaughnessy KD, et al. Targeted reinnervation for transhumeral amputees: current surgical technique and update on results. Plast Reconstr Surg. 2009;124(3):863-9.

24. Lipschutz RD, Kuiken TA, Miller LA, et al. Shoulder disarticulation externally powered prosthetic fitting following targeted muscle reinnervation for improved myoelectric control. J Prosthet Orthot. 2006;18(2):28-34.

25. Kuiken TA, Li G, Lock BA, et al. Targeted muscle reinnervation for real-time myoelectric control of multifunction artificial arms. JAMA. 2009;301(6):619-28.

26. Miller LA, Lipschutz RD, Stubblefield KA, et al. Control of a six degree of freedom prosthetic arm after targeted muscle reinnervation surgery. Arch Phys Med Rehabil. 2008;89(11):2057-65.

27. • Kuiken TA, Marasco PD, Lock BA, et al. Redirection of cutaneous sensation from the hand to the chest skin of human amputees with targeted reinnervation. Proc Natl Acad Sci USA. 2007;104(50):20061-6. This report details the near normal touch thresholds of reinnervated chest skin in TR subjects, and highlights that perceptual identity of the limb and chest is maintained separately even though there is a common skin surface.

28. Kuiken TA, Lowery MM, Stoykov NS. The effect of subcutaneous fat on myoelectric signal amplitude and cross-talk. Prosthet Orthot Int. 2003;27(1):48-54.

29. Schultz AE, Marasco PD, Kuiken TA. Vibrotactile detection thresholds for chest skin of amputees following targeted reinnervation surgery. Brain Res. 2009;1251:121-9.
30. •• Hebert JS, Olson JL, Morhart MJ, et al. Novel targeted sensory reinnervation technique to restore functional hand sensation after transhumeral amputation. IEEE Trans Neural Syst Rehabil Eng (in press). This paper is the first report of the fascicular end-toend targeted sensory reinnervation technique.

31. - MacDonald DB et al. Intraoperative monitoring using somatosensory evoked potentials - a position statement by the American Society of Neurophysiological Monitoring. Clin Neurophysiol. 2013;124(12):2291-316. doi:10.1016/j.clinph.2013.07.025. Accessed 1 Sept 2013. This statement provides detailed guidelines on the use of SSEP for monitoring the function of spine, brain, and peripheral nerves during surgical procedures.

32. Mahla ME, Long DM, McKennett J, et al. Detection of brachial plexus dysfunction by somatosensory evoked potential monitoring - a report of two cases. Anesthesiology. 1984;60(3):248-52.

33. Pitman MI, Nainzadeh N, Ergas E, Springer S. The use of somatosensory evoked potentials for detection of neuropraxia during shoulder arthroscopy. Arthroscopy. 1988;4(4):250-5.

34. Prielipp RC, Morell RC, Walker FO, et al. Ulnar nerve pressure: influence of arm position and relationship to somatosensory evoked potentials. Anesthesiology. 1999;91(2):345-54.

35. Salengros JC, Pandin P, Schuind F, Vandesteene A. Intraoperative somatosensory evoked potentials to facilitate peripheral nerve release. Can J Anaesth. 2006;53(1):40-5.

36. Gu B, Xie F, Jiang H, et al. Repair of electrically injured median nerve with the aid of somatosensory evoked potential. Microsurgery. 2009;29(6):449-55.

37. Triepel CR, Koman LA. Intraoperative electrophysiologic aid to nerve repair. Oper Tech Orthop. 2004;14(3):179-83.

38. Slutsky DJ. A practical approach to nerve grafting in the upper extremity. Atlas Hand Clin. 2005;10:73-92.

39. Hallin RG. Microneurography in relation to intraneural topography: somatotopic organisation of median nerve fascicles in humans. J Neurol Neurosurg Psychiatry. 1990;53(9):736-44.

40. Chow JA, Van Beek AL, Bilos ZJ, et al. Anatomical basis for repair of ulnar and median nerves in the distal part of the forearm by group fascicular suture and nerve-grafting. J Bone Joint Surg Am. 1986;68(2):273-80.

41. Apaydin N, Tubbs RS, Loukas M, Duparc F. Review of the surgical anatomy of the axillary nerve and the anatomic basis of its iatrogenic and traumatic injury. Surg Radiol Anat. 2009; 32(2):193-201.

42. Uz A, Apaydin N, Bozkurt M, Elhan A. The anatomic branch pattern of the axillary nerve. J Shoulder Elbow Surg. 2007;16(2):240-4.

43. Loukas M, Hullett J, Louis RG Jr, et al. The gross anatomy of the extrathoracic course of the intercostobrachial nerve. Clin Anat. 2006;19(2):106-11.

44. Pannucci C, Myckatyn TM, Mackinnon SE, Hayashi A. End-toside nerve repair: review of the literature. Restor Neurol Neurosci. 2007;25(1):45-63.

45. Tender GC, Kline DG. Anterior supraclavicular approach to the brachial plexus. Neurosurgery. 2006;58(4 Suppl 2):ONS-360-4.

46. Davies F, Gladstone RJ, Stibbe EP. The anatomy of the intercostal nerves. J Anat. 1932;66(Pt 3):323-33.

47. Sensinger JW, Schultz AE, Kuiken TA. Examination of force discrimination in human upper limb amputees with reinnervated limb sensation following peripheral nerve transfer. IEEE Trans Neural Syst Rehabil Eng. 2009;17(5):438-44.

48. Marasco PD, Schultz AE, Kuiken TA. Sensory capacity of reinnervated skin after redirection of amputated upper limb nerves to the chest. Brain. 2009;132(Pt 6):1441-8.

49. Kim DH, Hudson AR, Kline DG. Atlas of peripheral nerve surgery. 2nd ed. Philadelphia: Elsevier Saunders; 2013.

50. Kim K, Colgate JE. Haptic feedback enhances grip force control of sEMG-controlled prosthetic hands in targeted reinnervation amputees. IEEE Trans Neural Syst Rehabil Eng. 2012;20(6):798-805. 
51. Dawson MR, Fahimi F, Carey JP. The development of a myoelectric training tool for above-elbow amputees. Open Biomed Eng J. 2012;6:5-15.

52. Hernandez-Arieta A, Dermitzakis K, Damian D, et al. Sensorymotor coupling in rehabilitation robotics. In: Takahashi Y, editor. Service robot applications. Rijeka: InTech Europe; 2008. p. 21-36.

53. - Isaacs J. Major peripheral nerve injuries. Hand Clin. 2013;29(3):371-82. This review discusses epineurial, fascicular, group fascicular, and conduit repairs, and describes the sensory outcomes of various techniques.
54. Calvin-Figuière $S$, Romaiguère $P$, Roll JP. Relations between the directions of vibration-induced kinesthetic illusions and the pattern of activation of antagonist muscles. Brain Res. 2000;881(2):128-38.

55. - Marasco PD, Kim K, Colgate JE, et al. Robotic touch shifts perception of embodiment to a prosthesis in targeted reinnervation amputees. Brain. 2011;134(Pt 3):747-58. This paper demonstrates that physiologically appropriate cutaneous feedback from a prosthetic limb drives a perceptual shift towards embodiment of the device, which may help amputees more effectively incorporate an artificial limb as an integrated body part. 